\title{
A Eficiência de Cross-hedge do Risco de Preço de Frangos com o Uso de Contratos Futuros de Milho da BM\&F-BOVESPA
}

\author{
The Efficiency of Poultry Price Risk Cross-hedge with the Usage of Corn \\ Futures Contracts of BM\&F-BOVESPA
}

\author{
Waldemar Antonio da Rocha de Souza \\ Doutor em Economia Aplicada - USP/ESALQ \\ Professor Adjunto - UFAM \\ Av. Gal. Rodrigo Otávio Jordão Ramos, 3.000 - Coroado I, Manaus, AM - 69077-000 \\ warsouza@ufam.edu.br \\ Débora Fernandes Bellinghini \\ Mestranda em Economia Aplicada - USP/ESALQ \\ Av. Pádua Dias, 11 - Piracicaba, SP - 13418-900 \\ debora.bellinghini@gmail.com \\ João Gomes Martines-Filho \\ $P h D$ em Economia Agrícola - Ohio State University \\ Professor Doutor - USP/ESALQ \\ Av. Pádua Dias, 11 - Piracicaba, SP - 13418-900 \\ martines@usp.br \\ Pedro Valentim Marques \\ $P h D$ em Economia Agrícola - University of Kentucky \\ Professor Titular - USP/ESALQ \\ Av. Pádua Dias, 11 - Piracicaba, SP - 13418-900 \\ pvmarque@esalq.usp.br
}

\section{Resumo}

A indústria de frango apresenta risco de preço cuja análise e mitigação constituem etapas relevantes na administração eficiente das unidades produtivas, permitindo a alocação mais eficiente de recursos. Entretanto inexiste um contrato futuro específico para a avicultura no mercado futuro brasileiro, existindo, porém, um contrato futuro para milho na BM\&FBOVESPA. Dada a expressiva participação cruzada entre as indústrias de frango e de milho no Brasil, cabe indagar se o risco de preço do frango pode ser neutralizado com os contratos futuros domésticos de milho, analisando-se a eficiência do uso dos contratos futuros de milho negociados na BM\&F-BOVESPA em operações de cross-hedge do risco do preço de frangos. A questão de pesquisa tratada é: os contratos futuros de milho negociados na BM\&FBOVESPA fornecem mitigação eficiente de risco de preço para a indústria de frango nacional. As questões de análise são: i. quais as propriedades estatísticas das séries temporais dos preços a vista de frango e futuros de milho; ii. como obter diferentes níveis de crosshedge entre os preços de frango e de milho usando modelos alternativos de hedge: variânciamínima, sem hedge (no hedge), hedge total (naïve) e dinâmico; e, iii. qual a efetividade do cross-hedge obtido através do uso dos diversos modelos. Observa-se a baixa efetividade

Artigo publicado anteriormente nos Anais do II Congresso Nacional de Administração e Ciências Contábeis AdCont 2011 em 10/2011.

Artigo submetido em 17 de outubro de 2011 e aceito em 30 de novembro de 2011 pelo Editor Marcelo Alvaro da Silva Macedo, após double blind review. 
dessas operações sob diversos contextos de hedge e portfólio: ótimo, total, dinâmico. Os resultados podem ser atribuídos a estruturas microeconômicas distintas, como a baixa correlação entre as séries dos preços devido às especificidades de ambas as indústrias, a sazonalidade, os procedimentos de comercialização e a formação dos preços em ambos os mercados. Entretanto a robustez do mercado sinaliza potencial econômico-financeiro para a introdução de um contrato futuro de carne de frango no Brasil.

Palavras-chave: Risco de Preço. Mitigação. Contrato Futuro. Frango. Milho.

\begin{abstract}
The poultry industry bears price risk for which the analysis and mitigation are relevant steps to the efficient management of production units, allowing a more efficient allocation of resources. However a specific futures contract for the Brazilian poultry industry in the futures market does not exist, although there is a futures contract for corn is BM\&F-BOVESPA. Given the significant cross participation among the poultry and corn industries in Brazil, it is worth asking if the poultry price risk can be neutralized with the domestic corn futures, analyzing the efficiency of the use of corn futures contracts traded on BM\&F-BOVESPA in cross-hedge operations of the poultry price risk. The research question addressed is: corn futures contracts traded on the BM \& F BOVESPA, provide efficient mitigation of price risk for the national poultry industry. The survey questions are: i. what are the statistical properties of poultry spot prices and corn futures time series of, ii. how to get different cross-hedging levels of poultry prices and corn using alternative models of hedging: minimum-variance, no hedge, total hedge (naïve) and dynamic, and, iii. what are the cross-hedge effectiveness obtained through the use of the various models. The low effectiveness of these operations in different contexts and hedge portfolio: fine, full, dynamic is observed. The results can be attributed to different microeconomic structures, such as the low correlation between the price series due to the specificities in both industries, seasonality, marketing and pricing procedures in both markets. However, the strong market signals economic and financial potential for the introduction of a poultry future contract in Brazil.
\end{abstract}

Keywords: Price Risk. Mitigation. Future Contract. Poultry. Corn.

\title{
1. Introdução
}

Nas últimas três décadas, o agronegócio brasileiro desempenhou papel fundamental na geração de divisas para o país e no desenvolvimento econômico. Com o crescimento do porte, da competitividade e da complexidade das atividades agrícolas nos últimos anos, a informação transformou-se em uma ferramenta essencial para a tomada de decisões, tanto na etapa da produção, quanto na comercialização.

Dentre os setores de maior crescimento do agronegócio brasileiro, destaca-se a avilcultura, que apresentou forte evolução em períodos recentes, com base na evolução do faturamento, consumo per capita, produção e exportações.

O Brasil é o terceiro produtor mundial de aves, sendo o primeiro no ranking mundial de exportadores de carne de frango pelo terceiro ano consecutivo. A receita proveniente das exportações de carne de aves é a terceira maior na pauta do agronegócio brasileiro e a sexta na pauta geral. Na atual conjuntura, o Brasil embarca carne avícola para todos os continentes, abrangendo 146 países (LOPES; 2009).

O forte crescimento da indústria de frango brasileira impactou a demanda por milho. No período entre 2001 e 2007, a avicultura apresentou incremento de 89,3\% no consumo de milho, usado como ração animal (CALDARELLI, 2010). 
A indústria de frango apresenta risco de preço, similarmente aos demais produtos agropecuários. A análise do risco de preço e a sua mitigação constituem etapa relevante na administração eficiente das unidades produtivas, permitindo a alocação mais eficiente de recursos.

Entretanto inexiste um contrato futuro específico para a avicultura no mercado futuro brasileiro, havendo um contrato futuro para milho, negociado na BM\&F-BOVESPA. Dada a expressiva participação cruzada entre as indústrias de frango e de milho no Brasil, cabe indagar se o risco de preço do frango pode ser mitigado com contratos futuros domésticos de milho.

Dessa maneira, a questão de pesquisa tratada neste artigo é: os contratos futuros de milho negociados na BM\&F-BOVESPA fornecem mitigação eficiente de risco de preço para a indústria de frango nacional.

As questões de análise são: i. quais as propriedades estatísticas das séries temporais dos preços a vista de frango e futuros de milho; ii. como obter diferentes níveis de crosshedge entre os preços de frango e de milho usando modelos alternativos de hedge: variânciamínima, sem hedge (no hedge), hedge total (nä̈ve) e dinâmico; e, iii. qual a efetividade do cross-hedge obtido através do uso dos diversos modelos.

$\mathrm{O}$ artigo divide-se em cinco seções. Na primeira faz-se o levantamento da literatura sobre hedge e cross-hedge no Brasil e no exterior. Na segunda explicitam-se os modelos de hedge e cross-hedge, além dos dados utilizados. Apresentam-se os resultados na penúltima, resumindo e concluindo na última seção.

\section{Fundamentação Teórica}

A literatura acerca de estratégias de hedge e uso de mercados futuros, investiga a razão ótima de hedge, ou seja, a razão entre a posição nos mercados físicos e futuros de um agente que minimize sua posição de risco de preço no mercado.

$\mathrm{O}$ hedge é descrito na literatura de mercado futuro como o mecanismo existente entre os agentes participantes que visam transferir riscos de preços entre si. Quando um determinado agente se protege contra o risco de instabilidade de preços, fica sujeito somente à instabilidade da base, ou risco de base, que é a instabilidade existente entre a diferença de preços físicos, em determinada praça de comercialização e os preços futuros, tendo como referência o ponto de formação de preço (LEUTHOLD, 1989).

Working (1953) descreve o hedging como um contrato para comprar ou vender, em termos padronizados, estabelecido e supervisionado por uma bolsa de commodities, como um substituto temporário para um contrato posterior para comprar ou vender em outros termos. Segundo o autor, as motivações para fazer operações de hedge são: i. facilitar as decisões de compra e venda; ii. proporcionar mais liberdade para as decisões empresariais; iii. dispor de base confiável para efetuar o armazenamento de excessos de oferta de commodities; e, iv. reduzir os riscos empresariais.

Ederington (1979) conceitua o hedge como o ato de transferir o risco de preço de operadores de commodities para especuladores mais desejosos de assumir esse tipo de risco. Utiliza a teória de portfólios para explicar as operações de hedge em termos de maximização de retornos esperados e minimização de risco, inclusive em operações de cross-hedge.

Um modelo geral para cálculo de hedge ótimo, considerando as informações condicionais apropriadas, é desenvolvido por Myers e Thompson (1989). O uso de regressões simples entre as primeiras diferenças do nível dos preços a vista (variável dependente) e dos preços futuros (variável explicativa) geram os resultados de hedge mais compatíveis com o modelo geral.

A Eficiência de Cross-hedge do Risco de Preço de Frangos com o Uso de Contratos Futuros de Milho... 
Lence (1996) analisa o hedge de variância mínima relaxando algumas hipóteses básicas, a fim de obter parâmetros comportamentais mais condizentes com a realidade econômica dos agentes. Considerando os custos diretos das operações de hedge, dentre outros fatores, o resultado final é ínfimo, o que ajuda a explicar a razão do nível reduzido de utilização de contratos futuros diretamente por fazendeiros.

Brorsen (1995) deriva um modelo de hedge ótimo sob as hipóteses de neutralidade de risco e custos de empréstimos não-lineares. Conclui que as empresas mais alavancadas tendem a fazer mais hedge do que as não-alavancadas, quando se computam os custos operacionais do hedge.

Em Anderson e Danthine (1981) deduz-se um modelo de cross-hedging a partir da hipótese de média-variância de portfólios de agentes individuais, escolhendo-se os melhores contratos futuros disponíveis para hedging da posição a vista. Considera-se a existência de padronização dos contratos futuros e de diferenciação de produtos.

Dahlgran (2000) avalia a estratégia de cross-hedging de risco de preço de derivados de algodão construindo diversos portfólios que incluem vários tipos de contratos futuros. Apesar de eficiências de hedging estatisticamente significativas, há necessidade de avaliar os custos financeiros diretos e os custos indiretos das operações de hedge.

Analisa-se o cross-hedge pela avaliação do risco de base de diversos subprodutos suínos usando contratos futuros de suínos vivos, como instrumentos de administração do risco de preços ao atacado, em Hayenga e DiPietre (1982). Simulam-se diversas estratégias de uso de contratos futuros de suínos vivos na mitigação do risco de preço dos subprodutos suínos, a partir da abordagem de portfólio.

Foster e Whiteman (2002) desenvolvem um modelo para cross-hedge de futuros de soja através de abordagem bayesiana preliminar. A modelagem incorpora o risco de estimação, através de diversas especificações das séries temporais, p.ex., com e sem fatores sazonais, em logaritmo ou nível, usando informações de diversos mercados a vista e considerando as inovações na correlação entre o mercado a vista e a base, dentre outros fatores.

No Brasil, algumas abordagens exploram a temática de hedge ótimo. Silva et al (2003) analisam a efetividade do hedge de óleo, farelo e grão de soja na CBOT e BM\&F, encontrando que uma estratégia de cross-hedging com contratos futuros do grão na BM\&F possui baixa efetividade para óleo e farelo, sendo os contratos do tipo na CBOT os de melhores resultados.

Santos et al. (2008) investigaram o hedge de mínima variância na BM\&F para soja em grãos no Centro-Oeste, entre outubro de 2002 e dezembro de 2005 , encontrando que $44 \%$ da produção física dos produtores de soja de Goiás poderiam ser negociadas para reduzir o risco do preço da soja em $35 \%$.

Por sua vez, Martins e Aguiar (2004) analisam quais os períodos contratuais dos futuros na CBOT possuem a maior efetividade do hedge de soja em grão brasileira, concluindo que os de vencimento no segundo semestre, em especial julho e agosto, são os mais efetivos. Além disso, a efetividade é maior nas regiões próximas aos portos de exportação, em especial para São Paulo e Paraná.

Silveira (2002) analisa as operações de cross-hedge de bezerro com contratos futuros da $\mathrm{BM} \& \mathrm{~F}$ para diversas praças brasileiras. Calcula o risco de base, a razão ótima de crosshedge e a efetividade das operações. Conclui que as operações de cross-hedge não são eficientes para mitigar o risco de preço de bezerro.

A contribuição do presente estudo dá-se pela análise de operações de cross-hedge para a industria de frango nacional, com contratos futuros domésticos de milho negociados na BM\&F-BOVESPA, abordagem inédita na literatura sobre hedge no Brasil. 


\section{Metodologia e Dados}

A seguir explicitam-se o modelo teórico de cross-hedging, as metodologias de cálculo das razões de hedge: ótimo, total e dinâmico, bem como a explicitação dos dados utilizados.

\subsection{O Modelo Teórico de Cross-Hedging}

Conforme Pennacchi (2008) considera-se um modelo uniperiódico de um agente individual ou instituição que necessita comprar ou vender uma commodity no futuro e gostaria de fazer hedge do risco dessa operação assumindo posições no mercado futuro (ou em outro ativo financeiro).

Supondo que esse operador financeiro está comprometido no início do período, data 0 , a comprar y unidades de uma commodity com risco no final do período, data 1 , ao preço a vista então prevalecente $p_{1}$. Por exemplo, um compromisso de compra pode surgir se a commodity é um input necessário no processo de produção do operador, como uma usina de geração elétrica que utilize óleo diesel.

De maneira inversa, $y<0$ representa um compromisso de vender $-y$ unidades de uma commodity, a qual o operador produz e é não-estocável. Destaca-se que na data $0, y$ é determinística, enquanto $p_{1}$ é estocástico.

Existem $n$ ativos financeiros (p.ex., contratos futuros) na economia. Denota-se o preço do i-ésimo ativo financeiro na data $O$ por $p_{i 0}^{s}$. Seu preço na data $l$ é $p^{s}{ }_{i 1}$, o qual é incerto na data 0 . Seja $s_{i}$ a quantidade do i-ésimo ativo adquirido na data 0 . Portanto, $s_{i}<0$ indica uma posição vendida no ativo.

Definindo os vetores de preço e quantidade $n x l, \quad s \equiv\left[s_{1} \ldots s_{n}\right], \quad p_{0}^{s} \equiv\left[p_{10}^{s} \ldots p_{n 0}^{s}\right]$ e $p_{1}^{s} \equiv\left[p_{11}^{s} \ldots p_{n 1}^{s}\right]$. Também, define-se $p^{s} \equiv p_{1}^{s}-p_{0}^{s}$ como o vetor $n \times 1$ da variação de preços dos ativos. Este é o lucro da data 1 ao se assumir posições vendidas unitárias em cada um dos ativos (contratos futuros) na data 0 , tal que a lucratividade da posição de ativos do operador é $p^{s}{ }_{s}$. Também, define-se o primeiro e segundo momentos dos preços da data 1 da commodity a vista e do ativo financeiro: $E\left[p_{1}\right]=\bar{p}_{1}, \quad \operatorname{Var}\left[p_{1}\right]=\sigma_{00}, \quad E\left[p_{1}^{s}\right]=\bar{p}_{1}^{s}, \quad E\left[p^{s}\right]=\bar{p}^{s}$, $\operatorname{Cov}\left[p_{i 1}^{s}, p_{j 1}^{s}\right]=\sigma_{i j}, \operatorname{Cov}\left[p_{i 1}^{s}, p_{1}\right]=\sigma_{0 i}$ e a $(n+1) \mathrm{x}(n+1)$ matriz de covariância da commodity a vista e os ativos financeiros é dada por:

$$
\sum=\left[\begin{array}{cc}
\sigma_{00}, & \sum_{01} \\
\sum_{01} & \sum_{11}
\end{array}\right]
$$

Onde $\sum_{11}$ é uma matriz $n \times n$ cujo elemento i, j-ésimo é $s_{i j}$, e $\sum_{01}$ é um vetor 1 x $n$ cujo i-ésimo elemento é $s_{i}^{0}$.

Para simplificação, supõe-se que $y$ é fixo e, portanto, não é uma decisão variável na data 0 . Então, o lucro (riqueza) no final do período do operador financeiro, $W$, será dado por:

$$
W=p^{s^{\prime}}{ }_{s}-p_{1} y
$$

O que o operador deve decidir são as posições de ativos financeiros na data 0 . Supõese que o operador escolhe $s$ de forma a maximizar a seguinte função objetivo que depende linearmente da média e da variância do lucro: 


$$
\max _{s} E[W]-\frac{1}{2} \alpha \operatorname{Var}[W]
$$

Essa função objetivo resulta da maximização da utilidade esperada da riqueza quando os retornos de portfólio são normalmente distribuídos e a utilidade possui aversão absoluta ao risco. Substituindo na equação de lucro do operador, tem-se que:

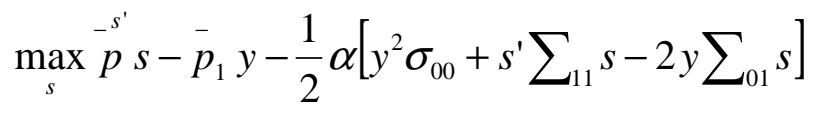

As condições de primeira ordem são:

$$
{ }^{-s}-\alpha\left[\sum_{11} s-y \sum_{01}^{\prime}\right]=0
$$

Portanto, as posições ótimas em ativos financeiros serão:

$$
\begin{gathered}
s=\frac{1}{\alpha} \sum_{11}^{-1^{-s}} p+y \sum_{11}^{-1} \sum_{01}^{\prime} \\
=\frac{1}{\alpha} \sum_{11}^{-1}\left(p_{1}^{-{ }^{-s}} p_{0}^{s}\right)+y \sum_{11}^{-1} \sum_{01}^{\prime}
\end{gathered}
$$

Considerando inicialmente o caso em que $y=0$. Isto pode ser analisado como a situação defrontada por um especulador puro, ou seja, um operador que não necessita fazer hedge. Se $n=1$ e $\stackrel{-s}{p_{1}}>p_{0}^{s}$, o especulador assume uma posição comprada no ativo financeiro, enquanto se ${ }^{-s} p_{1}<p_{0}^{s}$, o especulador assume uma posição vendida no ativo financeiro.

A magnitude da posição é ponderada pela volatilidade do ativo $\left(\sum_{11}^{-1}=1 / \sigma_{11}\right)$, e o nível de aversão ao risco do especulador, $\alpha$. Contudo, para o caso geral de $n>1$, um avanço ou declínio dos preços é insuficiente para determinar se um especulador assume uma posição comprada ou vendida no ativo particular. Todos os elementos de $\sum_{11}^{-1}$ precisam ser avaliados, uma vez que uma posição num determinado ativo pode trazer benefícios de diversificação.

Para o caso geral de $y \neq 0$, que é a situação defrontada por um hedger, a demanda para ativos financeiros é similar à de um especulador puro, ou seja, dependerá também das expectativas dos preços. Adicionalmente, existem componentes de hedging para a demanda por ativos financeiros, denominados $s^{h}$ :

$$
s^{h} \equiv y \sum_{11}^{-1} \sum_{01}^{\prime}
$$

Essa é a solução para o problema de $\min _{s} \operatorname{Var}(W)$. Assim, mesmo para um hedger, nunca é ótimo minimizar a volatilidade (risco) a menos que a aversão ao risco seja infinitamente grande. Mesmo um hedger avêsso a risco que maximize a utilidade esperada deve comportar-se de forma similar a um especulador, no que tange à importância do retorno esperado do ativo. Da definição acima, observa-se que quando $n=1$, a demanda por hedging pura por unidade da commodity adquirida, $s^{h} / y$, simplifica-se para:

Souza, W. A. R.; Bellinghini, D. F.; Martines-Filho, J. G.; Marques, P. V. 


$$
\frac{s^{h}}{y}=\frac{\operatorname{Cov}\left(p_{1}, p_{1}^{s}\right)}{\operatorname{Var}\left(p_{1}^{s}\right)}
$$

Para o caso geral, $n>1$, os elementos do vetor $\sum_{11}^{-1} \sum_{01}^{\prime}$ são iguais aos coeficientes $\beta_{1}$, ..., $\beta_{\mathrm{n}}$ do modelo de regressão múltipla:

$$
\Delta p_{1}=\beta_{0}+\beta_{1} \Delta p_{1}^{s}+\beta_{2} \Delta p_{2}^{s}+\ldots+\beta_{n} \Delta p_{n 1}^{s}+\varepsilon
$$

Onde $\Delta p_{1} \equiv p_{1}-p_{0}, \Delta p_{i}^{s} \equiv p_{1 i}^{s}-p_{0 i}^{i}$, e $\varepsilon$ um termo de erro de média zero.

Uma implicação da equação acima é que um trader poderá estimar as razões de hedge, $s^{h} / y$, fazendo uma regressão estatística usando uma série temporal histórica de $n \times 1$ vetores de variações de preços dos ativos. De fato, essa é a maneira padronizada do cálculo das razões de hedge na prática.

\subsection{As Razões de Hedge: Ótimo, Total e Dinâmico}

Conforme Souza et al. (2010), consideram-se duas metodologias para o cálculo da razão ótima de hedge do risco de preço do frango através dos contratos futuro de milho na $\mathrm{BM} \& \mathrm{~F}$ em determinado período de tempo.

O primeiro método considerado foi o da regressão linear simples, o qual baseia-se na hipótese de que a matriz de covariâncias é constante. O segundo é o modelo GARCH BEKK para a variância, que considera a dependência temporal da matriz. O teste de raiz unitária é utilizado para verificar a hipótese de estacionariedade da razão ótima de hedge encontrada neste último.

\subsubsection{Modelo de Hedge de Mínima Variância}

Segundo Hull (2008) o valor de hedge ótimo é aquele que descreve a razão ótima entre a posição futura e física de um determinado agente que minimize a variância, ou ainda, que apresente o menor risco. Esse valor pode ser encontrado pela seguinte razão:

$$
\text { Hedge Ótimo }=\frac{\operatorname{COV}\left(\Delta S_{t}, \Delta F_{t}\right)}{\operatorname{Var}\left(\Delta F_{t}\right)}
$$

Definindo-se:

$\Delta S_{t}=$ Primeira diferença dos preços físicos

$\alpha, \beta=$ Parâmetros lineares do modelo

$\Delta F_{t}=$ Primeira diferença dos preços futuros

Leuthold (1989) mostra que esses valores são obtidos pela estimativa de mínimos quadrados de:

$$
\Delta S_{t}=\alpha+\beta \Delta F_{t}
$$

No modelo o coeficiente estimado $\beta$ mostra a proporção da produção que deve ser negociada em mercados futuros, e que apresentará menor variância, sendo denominado de taxa ótima de hedge de variância mínima. É obtido pela estimação MQO. 
Dados os testes padrões de uma regressão linear o valor do coeficiente de determinação ou $R^{2}$ tem interpretação importante neste caso, refletindo a efetividade de proteção ocorrida, diminuindo a variância dos preços da posição total do agente (mercado físico mais futuro).

Entretanto, essa metodologia para cálculo da taxa de hedge ótimo deve ser vista com limitações, pois existem evidências, como a existência de correlação serial e heterocedasticidade, de que as taxas são dependentes da distribuição condicional das variações de preço da commodity, os quais se alterarão no tempo com forte grau de probabilidade, quando a distribuição condicional variar.

\subsubsection{Os Modelos ARCH, GARCH e GARCH-BEKK}

A literatura sobre preços agropecuários e séries financeiras geralmente aponta que essas séries são caracterizadas por apresentarem valores que oscilam consideravelmente, de um período para o outro, apresentando também erros de previsão pequenos em um período e consideravelmente grandes noutros. Esses fatos podem ser atribuídos principalmente à volatilidade desses mercados, sejam pelas mudanças nas políticas fiscal e monetária, seja por fatos relacionados à comercialização de dados produtos (CARTER, 1999).

Isto significa heterogeneidade da variância do erro de previsão, o que pode ser caracterizado como existência de autocorrelação dos erros de previsão. Assim, os erros de previsão de uma dada série são dependentes dos desvios da regressão. Esse comportamento que dá o caráter heterocedástico das variâncias dos erros de previsão caracteriza as séries de preços e financeiras em geral.

Em seu trabalho seminal Engle (1982), explora essa desigualdade existente na variância dos erros de previsão, em séries sujeitas à volatilidade. Para tanto, desenvolveu o que se chamam modelos auto-regressivos de heterocedasticidade condicional ARCH.

Esses modelos são definidos por Enders (2004), como utilizados para modelar séries que são não correlacionadas serialmente, entretanto, apresentam volatilidade, o que faz com que a variância condicional seja modelada como dependente dos valores passados da série, por meio de uma função quadrática. Em um modelo $\mathrm{ARCH}(1)$ a variância do erro $\varepsilon_{t}$ dependerá de uma constante mais o termo $\varepsilon_{t-1}^{2}$, essa é a principal característica dos modelos ARCH.

Generalizando, seja uma série $Y_{t}$, um modelo $\mathrm{ARCH}(\mathrm{r})$ pode ser definido como:

$$
\begin{aligned}
& Y_{t}=\beta_{0}+\beta_{1} X_{1 K}+\ldots+\beta_{k} X_{K t}+\varepsilon_{t} \\
& \operatorname{Var}\left(\varepsilon_{t}\right)=\sigma_{t}^{2}=\alpha_{0}+\alpha_{1} Y_{t-1}^{2}+\alpha_{2} Y_{t-2}^{2}+\ldots+\alpha_{r} Y_{t-r}^{2}
\end{aligned}
$$

Considerando as condições de estacionariedade de uma série temporal Morettin e Tolloi (2004) apontam que para que se tenha uma variância positiva e fracamente estacionária, para um modelo $\mathrm{ARCH}(\mathrm{r})$, as seguintes condições relativas aos coeficientes do modelo de variância dos erros têm que ser atendidas:

$$
\begin{aligned}
& \alpha_{0}>0, \alpha_{i}>0 \\
& \forall i=1,2,3 . . p \\
& \sum \alpha_{1}<1
\end{aligned}
$$


No que tange a $\varepsilon_{t}$, Engle (1982) supôs que esses erros são variáveis normais e com média zero e variância unitária, consideradas como uma seqüência de variáveis aleatórias independentes e identicamente distribuídas.

$\mathrm{O}$ uso de modelos ARCH em séries de preços (principalmente de commodities), assim como em séries financeiras é justificado na literatura pelo fato destas apresentarem-se não autocorrelacionadas. $\mathrm{O}$ escopo da abordagem dos modelos $\mathrm{ARCH}$ pode ser generalizado no que se chamam modelos auto-regressivos de heterocedasticia condicional generalizada (GARCH), os quais ampliam o conjunto de informações apresentado por uma série temporal, gerando uma formulação mais parcimoniosa, comparado a um modelo AR ou MA simplesmente (BOLLERSLEV, 1986).

Assim sendo, um modelo GARCH(r,m) é uma abordagem para modelar volatilidade, como um modelo $\mathrm{ARCH}(\mathrm{r})$, entretanto, com menos parâmetros. Trata-se de um modelo em que se tem a parte ARCH (representada por $\mathrm{r}$ ) e o componente GARCH (representado por $\mathrm{m}$ ).

A literatura recente apresenta argumentos que mostram que um modelo $\operatorname{GARCH}(1,1)$ é a especificação mais robusta verificada em aplicações de séries financeiras. Os trabalhos de Baba et al. (1990), Karolyi (1995) e recentemente de Yang e Allen (2004) apontam que um modelo GARCH $(1,1)$ por apresentar poucas restrições aos parâmetros são preferíveis aos modelos superparametrizados.

Um processo GARCH(1,1) pode ser descrito como:

$$
\begin{aligned}
& Y_{t}=\beta_{0}+\beta_{1} X_{1 t}+\ldots+\beta_{k t} X_{k t}+\varepsilon_{t} \\
& \operatorname{Var}\left(\varepsilon_{t}\right)=\sigma_{t}^{t}=\omega+\alpha_{1} Y_{t-1}^{2}+\beta_{1} \sigma_{t-1}^{2}
\end{aligned}
$$

Conforme Morettin e Toloi (2004), as condições de estacionariedade de um modelo GARCH(1,1), assim como a garantia de que a variância do processo seja positiva, podem ser resumidas em:

$$
\begin{aligned}
& \omega>0, \alpha_{1}>0 \\
& \beta_{1}>0 \\
& \alpha_{1}+\beta_{1}<1
\end{aligned}
$$

A estimação dos parâmetros dos modelos GARCH utilizados neste trabalho foi feita pelo método de máxima verossimilhança condicional, essa função é maximizada por métodos computacionais, através do modelo GARCH BEKK, proposto por Baba et al. (1990) a seguir formalizado, conforme explicitado em Bittencourt et al. (2006):

Definição: $\mathrm{O}$ modelo $\operatorname{BEKK}(\mathrm{p}, \mathrm{q}, \mathrm{k})$, com $\mathrm{H}_{\mathrm{t}}$ sendo a matriz de covariâncias condicionais, dado o conjunto de informações disponível em t, pode ser definido como:

$$
\begin{gathered}
\varepsilon_{t}=H_{t}^{\frac{1}{2}} \nu_{t} \\
H_{t}=C^{\prime} C+\sum_{i=1}^{q} A_{i}^{\prime} \varepsilon_{t-1} \varepsilon_{t-1}^{\prime} A_{i}+\sum_{j=1}^{p} B_{j}^{\prime} H_{t-j} B_{j}
\end{gathered}
$$

Onde C, A, B são matrizes de parâmetros $(\mathrm{K} \times \mathrm{K})$, com $\mathrm{K}=2$, no caso bivariado; C é uma matriz triangular superior; $\mathrm{p}$ e q são as ordens do modelo; e $\mathrm{k}$, o número de séries utilizadas.

Conforme Karolyi (1995), o modelo BEKK possui uma característica fundamental em sua especificação, qual seja, as suas configurações generalizadas, permitindo a influência cruzada entre as variâncias e as covariâncias condicionais dos índices de mercados, enquanto não implica a estimação de um grande número de parâmetros. 
No modelo BEKK, pode-se definir a razão ótima de hedge, quando o retorno é igualado à variação no logaritmo do preço da commodity, por:

$$
b_{t-1}=\operatorname{Cov}\left(\Delta p_{t}, \Delta f_{t} \mid \Omega_{t-1}\right) / \operatorname{Var}\left(\Delta f_{t} \mid \Omega_{t-1}\right)
$$

Onde $b_{t-1}$ indica a razão ótima de hedge e $p_{t}$ e $f_{t}$ indicam, respectivamente, os logaritmos dos preços à vista e futuros.

Conforme Baillie e Myers (1998) e Benninga et al. (1984), a minimização da variância supõe um alto grau de aversão ao risco. Entretanto, se os retornos esperados na operação de hedge forem zero, então a regra de mínima variância de hedge será a regra de máximautilidade esperada de hedge, generalizando a aplicabilidade da regra de mínima variância de hedge.

Dado o modelo bivariado de preços à vista e futuro, a razão ótima de hedge pode ser obtida através da matriz de covariância condicional $\mathrm{H}_{\mathrm{t}}$ e expressa por:

$$
\mathrm{b}_{\mathrm{t}-1}=\mathrm{h}_{21, \mathrm{t}} / \mathrm{h}_{22, \mathrm{t}}
$$

Onde $\mathrm{h}_{\mathrm{ij}, \mathrm{t}}$ é o elemento da i-ésima linha e j-ésima coluna da matriz de covariância condicional $\mathrm{H}_{\mathrm{t}}$. A razão ótima de hedge dinâmica, em estimativas amostrais, pode ser computada usando-se $\mathrm{H}_{\mathrm{t}}$, podendo ser representada sob a forma matricial por:

$$
\left[\begin{array}{ll}
\mathrm{h}_{11, \mathrm{t}} & \mathrm{h}_{12, \mathrm{t}} \\
\mathrm{h}_{21, \mathrm{t}} & \mathrm{h}_{22, \mathrm{t}}
\end{array}\right]=\left[\begin{array}{ll}
\mathrm{c}_{11} & 0 \\
& \\
\mathrm{c}_{21} & \mathrm{c}_{22}
\end{array}\right]+\left[\begin{array}{ll}
\mathrm{c}_{11} & \mathrm{c}_{21} \\
& \\
0 & \mathrm{c}_{22}
\end{array}\right]\left[\begin{array}{ll}
\mathrm{a}_{11} & \mathrm{a}_{12} \\
& \\
\mathrm{a}_{21} & \mathrm{a}_{22}
\end{array}\right]\left[\begin{array}{ll}
\varepsilon_{1, \mathrm{t}-1}^{2} & \varepsilon_{1, \mathrm{t}-1} \varepsilon_{2, \mathrm{t}-1} \\
& \\
\varepsilon_{2, \mathrm{t}-1} \varepsilon_{1, \mathrm{t}-1} & \varepsilon_{2, \mathrm{t}-1}^{2}
\end{array}\right]\left[\begin{array}{ll}
\mathrm{a}_{11} & \mathrm{a}_{12} \\
& \\
\mathrm{a}_{21} & \mathrm{a}_{22}
\end{array}\right]
$$

$$
+\left[\begin{array}{ll}
\mathrm{b}_{11} & \mathrm{~b}_{12} \\
\mathrm{~b}_{21} & \mathrm{~b}_{22}
\end{array}\right]\left[\begin{array}{ll}
\mathrm{h}_{11, \mathrm{t}-1} & \mathrm{~h}_{21, \mathrm{t}-1} \\
\mathrm{~h}_{21, \mathrm{t}-1} & \mathrm{~h}_{22, \mathrm{t}-1}
\end{array}\right]\left[\begin{array}{ll}
\mathrm{b}_{11} & \mathrm{~b}_{12} \\
\mathrm{~b}_{21} & \mathrm{~b}_{22}
\end{array}\right]
$$

\subsection{Dados}

Utilizam-se dois conjuntos de dados neste trabalho. O primeiro é a série de preços do frango semanal, em $\mathrm{R} \$ / \mathrm{kg}$, disponibilizada pelo Instituto de Economia Agrícola - IEA (http://www.iea.sp.gov.br/out/banco/menu.php). O segundo é a série de preços contínuos do contrato futuro de milho da BM\&F-BOVESPA, em valores semanais.

O período analisado é de Dezembro de 2006 a Dezembro de 2009, totalizando 156 observações semanais.

\section{Resultados e Discussão}

Explicita-se o gráfico no nível dos preços semanais do contrato futuro de milho da BM\&F-BOVESPA (PFMS) e a vista do frango (PSFS): 


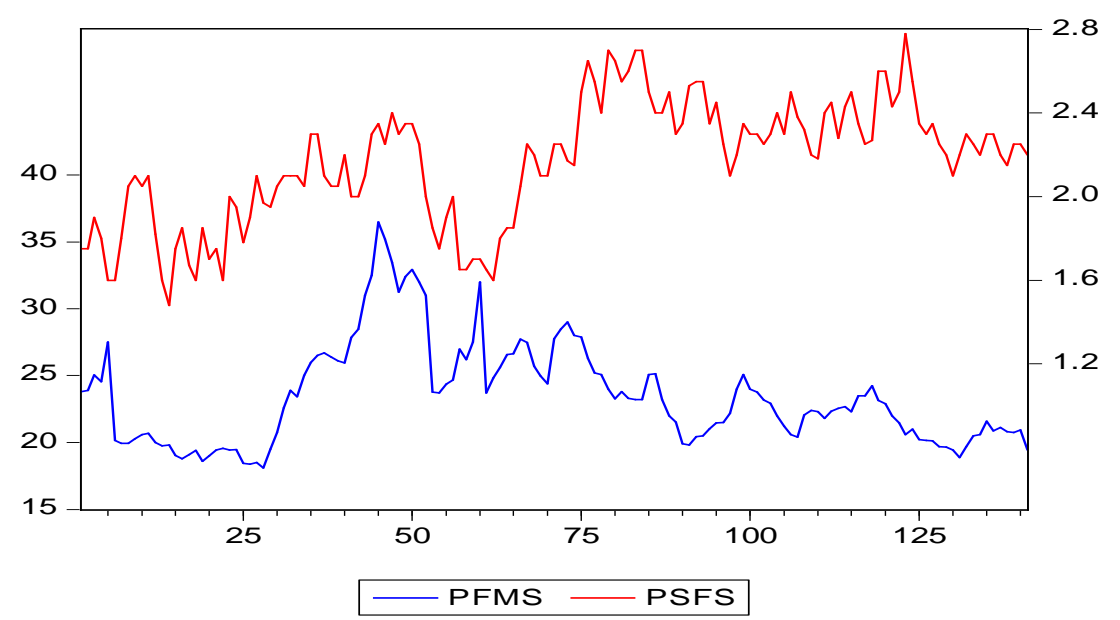

Gráfico 1 - Preços em nível - Futuro de milho PFMS e a vista de frango PSFS Em R\$ - Escalas: direita-PFMS, esquerda-PSFS

Nas Tabelas 1.1 e 1.2 explicitam-se os testes de raiz unitária, onde PFMS é o preço semanal do contrato futuro de milho e PSFS é o preço a vista semanal do frango:

Tab. 1.1 - Teste de Raiz Unitária (ADF) - Preço Futuro do Milho Semanal

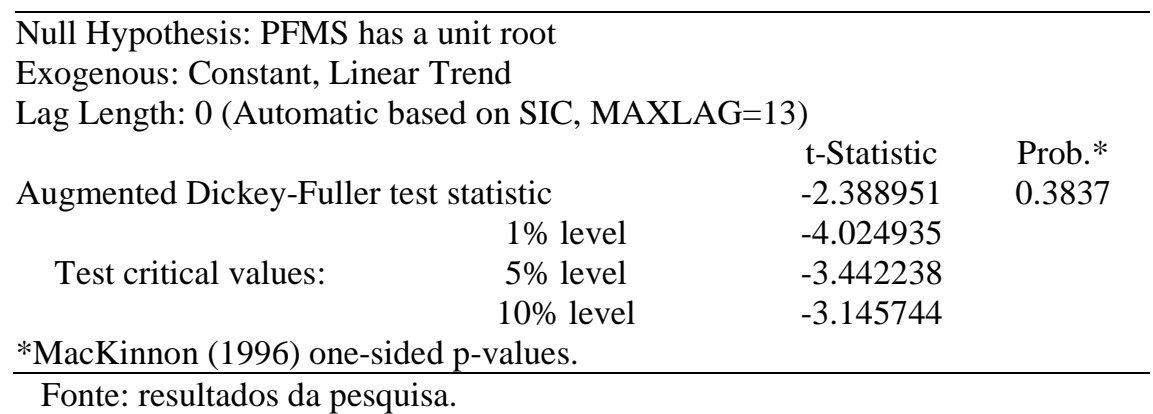

Tab. 1.2 - Teste de Raiz Unitária (ADF) - Preço A Vista do Frango Semanal

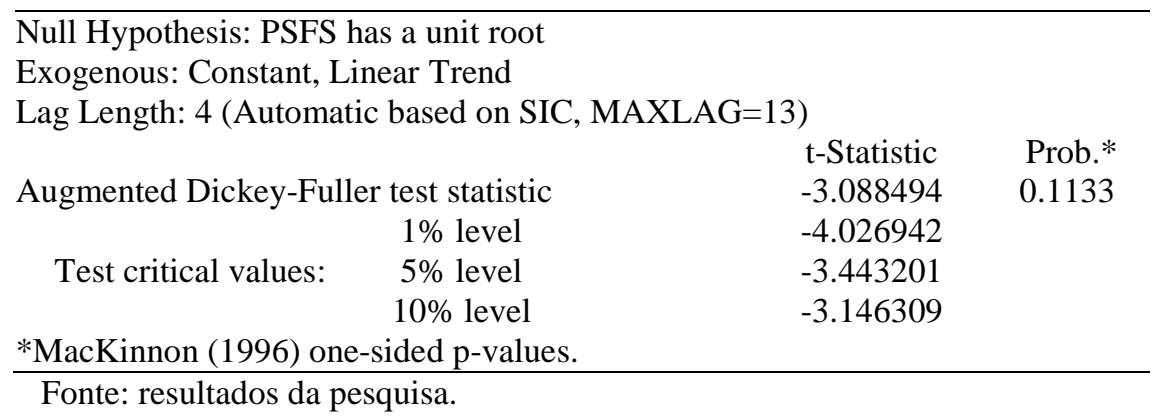

Conclui-se que ambas as séries possuem raiz unitária a 5\% de intervalo de confiança. Portanto a regressão simples deve ser feita entre as primeiras diferenças.

Explicita-se o cálculo da razão ótima de hedge, calculada pelo MQO das primeiras diferenças, sendo o preço a vista do frango a variável dependente e o preço futuro do milho a explicativa, Tabela 2: 
Tab. 2 - Razão de Hedge Ótimo - MQO

\begin{tabular}{lcccc}
\hline \begin{tabular}{l} 
Dependent Variable: DPSFS \\
Method: Least Squares \\
\multicolumn{1}{c}{ Variable }
\end{tabular}$\quad$ Coefficient & Std. Error & t-Statistic & Prob. \\
\multicolumn{1}{c}{ DPFMS } & 0.003305 & 0.011910 & 0.277498 & 0.7818 \\
R-squared & 0.002916 & 0.007689 & 0.379172 & 0.7051 \\
Adjusted R-squared & 0.001041 & Mean dependent var & 0.003214 \\
S.E. of regression & -0.006198 & S.D. dependent var & 0.140453 \\
Sum squared resid & 0.140887 & Akaike info criterion & -1.067528 \\
Log likelihood & 2.739200 & Schwarz criterion & -1.025505 \\
F-statistic & 76.72697 & Hannan-Quinn criter. & -1.050451 \\
Prob(F-statistic) & 0.143772 & Durbin-Watson stat & 2.059083 \\
\hline
\end{tabular}

Fonte: resultados da pesquisa.

Observa-se uma razão de hedge, equivalente ao cross-hedge de frango e milho, de $0,3 \%$, com efetividade de $0,1 \%$.

O cálculo do hedge dinâmico, modelo GARCH BEKK está na Tabela 3:

Tab. 3 - Hedge Dinâmico - 1.a Diferença preços a vista de frango e futuro de milho - semanal

\begin{tabular}{|c|c|c|c|c|}
\hline \multicolumn{5}{|c|}{$\begin{array}{l}\text { Estimation Method: ARCH Maximum Likelihood (Marquardt) } \\
\text { Covariance specification: Diagonal BEKK }\end{array}$} \\
\hline & Coefficient & Std. Error & z-Statistic & Prob. \\
\hline $\mathrm{C}(1)$ & -0.023497 & 0.092215 & -0.254 & 89 \\
\hline $\mathrm{C}(2)$ & 0.002777 & 0.011820 & 0.234926 & 0.8143 \\
\hline \multicolumn{5}{|c|}{ Variance Equation Coefficients } \\
\hline $\mathrm{C}(3)$ & 0.069496 & 0.065319 & 1.063943 & 0.2874 \\
\hline $\mathrm{C}(4)$ & 0.005636 & 0.021 & 0.263124 & 0.7925 \\
\hline $\mathrm{C}(5)$ & 0.505655 & 0.1 & 4.261725 & 0.0000 \\
\hline$C(6)$ & 0.050569 & 0.138147 & 0.366051 & 0.7143 \\
\hline $\mathrm{C}(7)$ & 0.867338 & 0.037045 & 23.41333 & 0.0000 \\
\hline $\mathrm{C}(8)$ & 0.843162 & 0.649647 & 1.297878 & 0.1943 \\
\hline Log likelihood & -159.4414 & \multicolumn{2}{|c|}{ Schwarz criterion } & 2.560114 \\
\hline Avg. log likelihood & -0.569434 & \multirow{2}{*}{\multicolumn{2}{|c|}{ Hannan-Quinn criter. }} & 2.460329 \\
\hline Akaike & .392020 & & & \\
\hline
\end{tabular}

Fonte: resultados da pesquisa.

A partir dos resultados da matriz de covariância condicional para cada período, obtêmse os portfólios correspondentes às razões de hedge dinâmico, cuja efetividade, dada pela redução de variância média, é de aproximadamente $2,0 \%$.

Para fins comparativos, explicita-se o Gráfico $2 \mathrm{com}$ as razões de hedge ótimo e dinâmico:

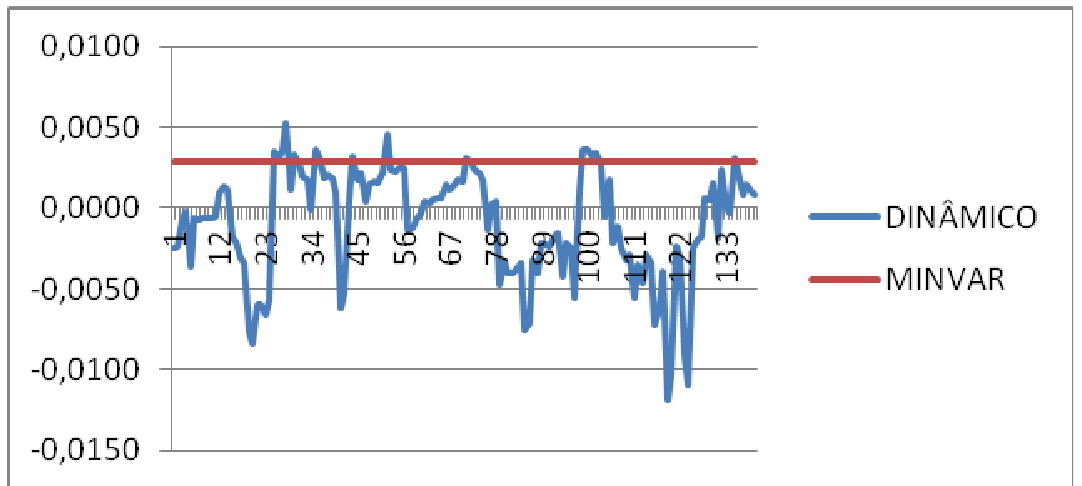

Gráfico 2 - Comparativo entre as Razões de Hedge de Mínima Variância e Dinâmico

Souza, W. A. R.; Bellinghini, D. F.; Martines-Filho, J. G.; Marques, P. V. 
Por último, calcula-se a efetividade do hedge total (naïve), cuja razão de hedge é de $100 \%$, ou seja, toda a posição a vista no mercado de frango é operada em igual valor no mercado futuro de milho. Neste caso, a efetividade do cross-hedge é de 0,24\%.

Observa-se a baixa efetividade do cross-hedge nas três abordagens analisadas: variância mínima, dinâmico e total (nä̈ve).

\section{Conclusões}

Neste estudo avalia-se a capacidade dos contratos futuros de milho negociados na BM\&F-BOVESPA em mitigarem de forma eficiente o risco de preço para a indústria de frango nacional, através de operações de cross-hedge.

A baixa efetividade do cross-hedge entre os preços a vista do frango e o preço futuro do milho na BM\&F-BOVESPA encontrada pode ser atribuída à baixa correlação entre os preços devido a estruturas microeconômicas distintas, como a sazonalidade das vendas, a formação de estoques, distribuição do ano-safra e condições de abate do plantel de aves.

Resultado similar de baixa efetividade de cross-hedge com contratos futuros no Brasil foi anteriormente evidenciado por Silveira (2002), analisando operações de cross-hedge de bezerro com futuros de boi gordo na BM\&F.

Entretanto, o mercado subjacente de frango a vista no Brasil é relevante na pauta do agronegócio. Tal fato é uma pré-condição para a avaliação da criação de mercado futuro de frango na BM\&F-BOVESPA. Como exemplos recentes pode-se citar of mercados futuros introduzidos no CME GROUP, nos EUA: Distillers' Dried Grain Futures (DDG) (subproduto de milho após destilação de etanol) e óleo de palma. No Brasil se observam os recentes contratos futuros de etanol e base de milho, na BM\&F-BOVESPA.

Como futuras pesquisas no assunto sugerem-se o estudo da viabilidade de um contrato futuro de frango para o Brasil, bem como o avanço das análises de efetividade de operações de cross-hedge com os contratos futuros atualmente existentes na BM\&F-BOVESPA, a correlação e volatilidade dos preços futuros nacionais, a formação de base nas diversas regiões do País, dentre as temáticas principais.

\section{Referências}

ANDERSON, R.W., DANTHINE, J.P. Cross hedge. Journal of Political Economy, v. 84, p. 1182-1196, 1981.

BABA, Y., ENGLE, R.F., KRAFT, D.F., KRONER, K.F. Multivariate simultaneous generalized ARCH. San Diego: University of California, mimeo, 1990.

BAILLIE, R.T., MYERS, R.J. Modeling commodity price distributions and estimating the optimal futures hedge. Working Paper. Nova Iorque: University of Columbia, 1998.

BITENCOURT, W.A, SILVA, W.S., SÁFADI, T. Hedge Dinâmicos: Uma Evidência para os Contratos Futuros Brasileiros. Organizações Rurais \& Agroindustriais, v. 8, n. 1, p. 71-78, 2006.

BOLLERSLEV, T. Generalized autoregressive conditional heteroscedasticity. Journal of Econometrics, v. 31, n. 3, p. 307-327, 1986. 
BRORSEN, B.W. Optimal hedge ratios with risk-neutral producers and nonlinear borrowing costs. American Journal of Agricultural Economics, v. 77, p. 174-181, 1995.

CALDARELLI, C.E. Fatores de influência no preço do milho no Brasil. Tese (Doutorado em Economia Aplicada). Escola Superior de Agricultura "Luiz de Queiroz", Universidade de São Paulo, Piracicaba, 2010.

CARTER, C.A. Commodity futures markets: a survey. Australian Journal of Agriculture and Resource Economics, v. 43, p. 209-247, 1999.

COLLINS, R.A. Toward a positive economic theory of hedging. American Journal of Agricultural Economics, v. 79, p. 488-499, 1997.

DAHLGRAN, R.A. Cross-Hedging the Cottonseed Crush: A Case Study. Agribusiness, v. 16, n. 2, p. 141-158, 2000.

EDERINGTON, L.H. The Hedging Performance of the New Futures Markets. The Journal of Finance, v. 34, n. 1, p. 157-170, 1979.

ENDERS, W. Applied econometric time series. 3 ed. Nova Iorque: John Wiley \& Sons, 2010, 317 p.

ENGLE, R.F. Autoregressive conditional heteroscedasticity with estimate of variance of U.K inflation. Econometrica, v. 50, p. 987-1008, 1982.

FOSTER, F.D., WHITEMAN, C.H. Bayesian cross-hedging: an example from the soybean market. Australian Journal of Management, v. 27, p. 95-122, 2002.

HAYENGA, M.L.,DIPIETRE, D.D. Cross-Hedging Wholesale Pork Products Using Live Hog Futures. American Journal of Agricultural Economics, v. 64, n. 4, p. 747-751, 1982.

HULL, J. Options Futures \& Other Derivatives. 8 ed. New Jersey: Prentice Hall, 2008, 698 p.

KAROLYI, G.A. A multivariate GARCH model of international transmissions of stock returns and volatility: the case of the United States and Canada. Journal of Business and Economic Statistics, v. 13, p. 11-25, 1995.

LENCE, S. Relaxing the Assumptions of Minimum-Variance Hedging. Journal of Agricultural and Resource Economics, v. 21, n. 1, p. 39-55, 1996.

LEUTHOLD, R.M., JUNKUS, J.C., CORDIER, J.E. The Theory and Practice of Futures Markets. Urbana: Stipes Publising L.L.C., 1989, 409 p.

LOPES, C.J.R. Estimação da equação de oferta de exportação da carne de frango brasileira - 1994 a 2008. Piracicaba, Monografia (Graduação) Escola Superior de Agricultura "Luiz de Queiroz", Universidade de São Paulo, 2009.

MARTINS, A.G., AGUIAR, D.R.D. Efetividade do hedge de soja em grão brasileira com contratos futuros de diferentes vencimentos na CHICAGO BOARD OF TRADE. Revista de Economia e Agronegócio, v. 2, n. 4, p. 449-472, 2004. 
MORETTIN, P. A., TOLOI, C.M.C. Análise de séries temporais. 2 ed. São Paulo: Edgard Blucher, 2004, $538 \mathrm{p}$.

MYERS, R.J., THOMPSON, S.R. Generalized Optimal Hedge Ratio Estimation. American Journal of Agricultural Economics, v.71, n. 4, p. 858-868, 1989.

PANELL, D.J., HAILU,G., WEERSINK, A., BURT, A. More reasons why farmers have so little interest in futures markets. Agricultural Economics, v. 39, p. 41-50, 2008.

PENNACCHI, G.C. Theory of Asset Pricing. Boston: Pearson Education, Inc., 2008, 457 p.

PENNINGS, J.M.E., MEULENBERG, M.T.G. Hedging Efficiency: A Futures Exchange Management Approach. The Journal of Futures Markets, v. 17, n. 5, p. 599-615, 1997.

PURCELL, W.D. Agricultural Futures and Options: Principles and Strategies. Nova Iorque: Macmillan, 1991, $363 \mathrm{p}$.

SANTOS, M.P., BOTELHO FILHO, F.B., ROCHA, C.H. Hedge de mínima variância na BM\&F para soja em grãos no Centro-Oeste. Revista da Sociedade e Desenvolvimento Rural, v. 1, n. 1, p. 203-211, 2007.

SHI, W., IRWIN, S.H. Optimal hedging with a subjective view: an empirical Bayesian approach. American Journal of Agricultural Economics, v. 87, p. 918-930, 2005.

SILVA, A.R.O. da, AGUIAR, D. R. D., LIMA, J. E. Hedging with futures contracts in the Brazilian soybean complex: BM\&F vs. CBOT. Revista de Economia e Sociologia Rural, v. 41, n. 2, p. 383-405, 2003.

SILVEIRA, R.L.F. Análise das Operações de Cross-hedge do Bezerro e do Hedge de Boi Gordo no Mercado Futuro. Dissertação (Mestrado). Escola Superior de Agricultura "Luiz de Queiroz". Universidade de São Paulo. Piracicaba, 2002.

SOUZA, W.A.R., CALDARELli, C.E., ROCHA, C.M., MARTINES-FILHO, J.M. The dynamic hedging effectiveness for soybean farmers of Mato Grosso with futures contracts of BM\&F. Organizações Rurais e Agroindustriais, v. 12, p. 1-21, 2010.

WORKING, H. Hedging Reconsidered. Journal of Farm Economics, v. 35, n. 4, p. 544-561, 1953.

YANG, W., ALLEN, D.E. Multivariate GARCH hedge ratios and hedging effectiveness in Australian futures markets. Accounting and Finance, v. 45, p.301-321, 2004,. 